\title{
Hierarchical and sensitivity analysis for noise abatement departure procedures
}

\author{
Xavier Prats* $\quad$ Joseba Quevedo ${ }^{\dagger} \quad$ Vicenç Puig ${ }^{\dagger}$ \\ Fatiha Nejjari ${ }^{\dagger}$ \\ Technical University of Catalonia (UPC)
}

\begin{abstract}
Nowadays, the establishment of new noise abatement procedures is one of the main issues that airport authorities have to address. As a continuation of previous studies, the authors present in this paper a strategy for designing such procedures aimed at reducing the global annoyance perceived for the population living around the airports. Noise optimal departing routes are computed for a specific aircraft type and for a given scenario. This non-linear multi-objective optimal control problem is solved by using lexicographic and hierarchical optimisation techniques. This approach establishes a hierarchical order among all the optimisation objectives, which in this work are set as the maximum perceived noise levels at different noise sensitive locations. If the prioritisation that should be adopted is not clear, this work presents two techniques aimed at obtaining the best solution according to an equitable principle, based on minimising the worst case noise annoyance. First approach requires a high computational cost, but the best trajectory is obtained, being the opposite case for the second technique, which is based on heuristics.
\end{abstract}

\section{Nomenclature}

$\begin{array}{ll}C_{a} & \text { airliner cost } \\ \vec{r} & \text { position vector } \\ \chi & \text { aerodynamic yaw angle } \\ e & \text { eastwards position } \\ \gamma & \text { aerodynamic pitch angle } \\ h & \text { height } \\ h_{c} & \text { thrust cutback height } \\ J_{i} & \text { objective function } i \\ \mu & \text { aerodynamic bank angle } \\ n & \text { northwards position } \\ n_{z} & \text { vertical load factor } \\ \vec{p} & \text { parameters vector } \\ \vec{x} & \text { state vector } \\ \vec{z} & \text { decision variables vector } \\ s & \text { procedure design gradient } \\ t & \text { time } \\ t_{0} & \text { initial time } \\ t_{f} & \text { final time } \\ \vec{u} & \text { control vector } \\ v & \text { true airspeed } \\ v_{2} & \text { minimum climb safe speed }\end{array}$

*EPSC/UPC. Avinguda del Canal Olmpic, 15. 08860 Castelldefels, Spain

${ }^{\dagger}$ ESAII. Rambla St. Nebridi 10. 08222 Terrassa, Spain. 


\section{Introduction}

The design of noise abatement procedures, aimed at reducing the noise exposure of the population around airports, is one of the main issues that airport authorities and national navigation services providers have to address. National navigation services providers are responsible for designing these kinds of procedures, taking into account several factors such as obstacle clearance, airport and air traffic management issues, separation criteria, etc. In addition, the aforementioned procedures could be designed to mitigate the aircraft noise over sensitive locations around the airports. In this context, the horizontal flight path may be designed to avoid over-flying sensible locations and the vertical path may be specified by scheduling different speed and/or thrust profiles.

The International Civil Aviation Organisation (ICAO) publishes two different Noise Abatement Departure Procedures (NADP). ${ }^{1}$ NADP are generic procedures and are far from being the optimum ones regarding noise minimisation. This is due to several factors, such as the impossibility to define a general procedure satisfying the specific problems that may affect each particular airport, air traffic management and airport capacity constraints or even the limitations of nowadays on-board technology. Nevertheless, some works in theoretical optimal trajectories, minimising the noise impact in departure or approaching procedures, are done at research level. In previous works, ${ }^{2,3}$ the authors presented a optimisation framework where noise optimal departing routes were computed for a specific aircraft type and for a given scenario. Similar approaches can be found in the literature, like for example the studies made by Visser et al., ${ }^{4-7}$ Atkins and Xue ${ }^{8,9}$ and Clarke et al. ${ }^{10,11}$ All the results and conclusions arisen from these works are encouraging and will set the basis for new noise abatement procedures, specially regarding the forthcoming of new navigation concepts, such as area navigation (RNAV) or Performance Based Navigation (PBN). These concepts will allow for air navigation procedures to be designed with a higher level of flexibility than conventional radionavigation ones. $^{12}$

A common issue when dealing with this kind of trajectory optimisation problems is that several criteria may be satisfied at the same time. These criteria, which are the noise or noise annoyance at different locations around the airport, are usually not compatible since the trajectory that optimise one criterion may be completely different from the trajectory optimising another one. Thus, one has to deal with multiobjective optimisation techniques in order to identify the absolute optimal trajectory among all the local ones by comparing a certain global performance index. In this work it is presented a strategy that uses lexicographic and hierarchical optimisation techniques aimed at finding the best trajectory minimising the noise at the worst sensitive location.

Section II of this paper presents briefly the optimisation problem that is considered. A multi-objective optimal control problem is formulated and, in section III the lexicographic and hierarchical optimisation algorithms are presented. Finally, section IV presents an application example of these optimisation techniques.

\section{Statement of the problem}

In this section, the optimisation problem is presented as well as all involved equations that define the optimisation criteria and constraints. For a more detailed description of this problem, please refer to Ref. 2 .

A given airport with its surrounding cartography, geography and meteorological data, defines a scenario that will be used to compute a given noise nuisance in function of the emitted aircraft noise along its trajectory. This value, together with some airliner economic considerations, will define one or several optimisation criteria. Then, an optimisation algorithm will compute the best departing or approaching trajectory minimising these criteria and satisfying a set of trajectory constraints which, in turn, will depend on the dynamics of the aircraft, navigation constraints and specific airspace configurations.

The optimisation process can be formally written as a constrained multi-objective optimal control problem in a given time interval. Let $\vec{x}(t) \in \mathbb{R}^{n_{x}}$ be the state vector describing the trajectory of the aircraft over the time $t, \vec{u}(t) \in \mathbb{R}^{n_{u}}$ the control vector that leads to a specific trajectory and $\vec{p} \in \mathbb{R}^{n_{p}}$ a set of control parameters not dependent on $t$. The goal is to find the best trajectory that minimises a given set of optimisation objectives (or criteria) $\vec{J} \in \mathbb{R}^{n_{j}}$. Namely:

$$
\min _{\vec{z} \in \mathcal{Z}} \vec{J}(\vec{z})=\min _{\vec{z} \in \mathcal{Z}}\left[J_{1}(\vec{z}), J_{2}(\vec{z}), \cdots, J_{n_{j}}(\vec{z})\right]
$$

where $\mathcal{Z} \subseteq \mathbb{R}^{n_{x}+n_{u}+n_{p}+1}$, is the admissible set of decision variables $\vec{z}=\left[\vec{x}(t), \vec{u}(t), \vec{p}, t_{f}\right]^{T}$, and $J_{i}(\vec{z})$ are 
scalar valued functions representing each individual criterion or objective.

\section{A. Optimisation criteria}

In this work, the maximum perceived sound level received at each given location $\left(L_{\max }\right)$ is used as noise nuisance criterion in the optimisation process. To compute noise functions the same methodology employed by the Integrated Noise Model (INM) ${ }^{13}$ program is implemented. In addition, airliner cost and Air Traffic Management (ATM) efficiency are taken into account when designing aircraft trajectories. In this context, Fuel and/or Time spent during the trajectory may be considered as optimisation objectives too. However, it would be incomplete to consider only these magnitudes regardless of the altitude achieved at the end of the procedure $h\left(t_{f}\right)$. Reaching a low final altitude would lead to small time or fuel consumption figures during the departure but the consumption would increase in the following phase, when trying to gain the altitude required to reach the optimal cruise flight level.

Current Flight Management and Guidance Systems (FMGS) equipping a wide number of aircraft deal with a compound cost function which involves fuel and time consumption during the flight. A cost index parameter $(C I)$ relates the cost of time delay to the price of the fuel and its value is carefully chosen by the operator prior to each flight. Following the same philosophy, an Height Index $(H I)$ is proposed in this work in order to take into account the final altitude achieved at the end of the departing trajectory. Then, an airliner cost compound function is defined as:

$$
C_{a}=\text { Fuel }+C I \cdot \text { Time }-H I \cdot h\left(t_{f}\right)
$$

where, by definition, $C I>0$ and $H I>0$.

\section{B. Constraints}

In order to guarantee a feasible and acceptable trajectory as a result of the optimisation process presented above, several constraints must be taken into account, leading to the definition of the admissible set of decision variables $\mathcal{Z}$.

\section{Dynamics of the aircraft}

Aircraft dynamics are described by a set of differential equations:

$$
\frac{\mathrm{d} \vec{x}(t)}{\mathrm{d} t}=\vec{f}[\vec{x}(t), \vec{u}(t), \vec{p}]
$$

where $\vec{f}$ contains the equations of motion corresponding to a general non-linear aircraft model. ${ }^{14}$ State vector is defined in this work as:

$$
\vec{x}(t)=[v(t) \chi(t) \gamma(t) n(t) e(t) h(t)]^{T}
$$

being $v(t)$ is the module of the relative air to aircraft velocity, also known as True Airspeed (TAS), $\chi(t)$ is the aerodynamic yaw angle and $\gamma(t)$ is the aerodynamic pitch angle, also known as Flight Path Angle $(F P A)$. Vector $\vec{r}(t)=[n(t) e(t) h(t)]^{T}$ represents the aircraft centre of mass position, being $n(t)$ and $e(t)$ the Northwards and Eastwards distances, respectively, from a arbitrary chosen origin and $h(t)$ the height above the runway.

On the other hand, the control vector is defined as:

$$
\vec{u}(t)=\left[n_{z}(t) \mu(t)\right]^{T}
$$

being $n_{z}(t)$ the vertical load factor and $\mu(t)$ the aerodynamic bank angle.

\section{Event constraints}

In a departure procedure, the horizontal final coordinates, namely $n\left(t_{f}\right)$ and $e\left(t_{f}\right)$, are fixed and chosen by the procedure designer. The altitude at this final point is not usually fixed, but is constrained to be higher than a minimum safe altitude $h_{f}^{\min }$ and lower than a maximum altitude $h_{f}^{\max }$, due to air traffic management criteria. 
The initial take-off phase going from ground level to a height $400 \mathrm{ft}$ will not be considered in the optimisation process since the standard operational regulations ${ }^{1,15}$ almost restrict all degrees of freedom during this particular phase. In this initial phase the aircraft follows a straight trajectory, following the departing runway heading, at a constant speed (usually $v_{2}$ or slightly above), which depends on the aerodynamics and the actual weight of the aircraft. For this problem, and for the sake of simplicity, initial horizontal coordinates are set to zero at the point where the aircraft reaches a height of $400 \mathrm{ft}$ above the runway. Moreover, during a normal take-off, the landing gear has been completely retracted when passing $400 \mathrm{ft}$ so it is not considered in the simulations. Taking all these considerations into account, end point constraints are fixed to known values as follows:

$$
\begin{array}{lll}
v\left(t_{0}\right)=v_{2} & \chi\left(t_{0}\right)=\chi_{R W Y} & \gamma\left(t_{0}\right)=\gamma_{2} \\
n\left(t_{0}\right)=0 & e\left(t_{0}\right)=0 & h\left(t_{0}\right)=400 \mathrm{ft} \\
n\left(t_{f}\right)=n_{f} & e\left(t_{f}\right)=e_{f} & h_{f}^{\min } \leq h\left(t_{f}\right) \leq h_{f}^{\max }
\end{array}
$$

Here, $\gamma_{2}$ is the flight path angle that results when the aircraft is flying at $v_{2}$ speed while applying TakeoffGo Around thrust settings.

\section{Path and Box constraints}

Due to ATM reasons, a departing trajectory must be contained inside a specific airspace area in order to guarantee a safe minimum separation with other existing procedures in the area. Mathematically speaking, the area of admissible trajectories can be defined by:

$$
\vec{\psi}_{L} \leq \vec{\psi}(\vec{x}(t)) \leq \vec{\psi}_{U}
$$

Functions $\vec{\psi}$ and bounds $\vec{\psi}_{L}$ and $\vec{\psi}_{U}$ represent all the possible restrictions in the airspace where the departure takes place.

In addition, and for operational reasons, it is enforced that the speed and altitude of the aircraft should not decrease during the whole departure procedure. Moreover, a minimum procedure design gradient $(s)$ must be guaranteed. ICAO Document $8168^{1}$ specifies a default gradient of $s=3.3 \%$, which can be higher for obstacle clearance reasons. The value of $s$ will be chosen in function of the scenario and the aircraft operator. Namely:

$$
\frac{\mathrm{d} v}{\mathrm{~d} t} \geq 0 ; \quad \frac{\mathrm{d} h}{\mathrm{~d} t} \geq 0 ; \quad h \geq h\left(t_{0}\right)+s \sqrt{n^{2}+e^{2}}
$$

Finally, some state and control variables should be bounded in order to ensure existing operational or safety requirements for a given aircraft. Therefore, the following constraints are defined:

$$
\begin{aligned}
v_{2} & \leq v(t) \leq v_{\max } \\
0.85 \leq n_{z}(t) & \leq 1.15 \\
-25^{\circ} \leq \mu(t) & \leq 25^{\circ} \\
244 \mathrm{~m} \leq h_{c} & \leq 1000 \mathrm{~m}
\end{aligned}
$$

The load factor $\left(n_{z}\right)$, bank angle $(\mu)$ and maximum thrust cutback height $\left(h_{c}\right)$ bound values correspond to typical values applied by aircraft operators. For noise abatement, the thrust cutback height should be greater than $800 \mathrm{ft} .^{1}$

\section{Numerical solution of the optimisation problem}

The optimal control problem described above, which contains differential and algebraic constraints, is transformed in two steps into a non linear programming (NLP) problem with only algebraic constraints. First, differential equations (3) are written in its equivalent integral form:

$$
\vec{x}(t)=\vec{x}\left(t_{0}\right)+\int_{t_{0}}^{t} \vec{f}(\vec{x}(\tau), \vec{u}(\tau), \vec{p}) \mathrm{d} \tau
$$




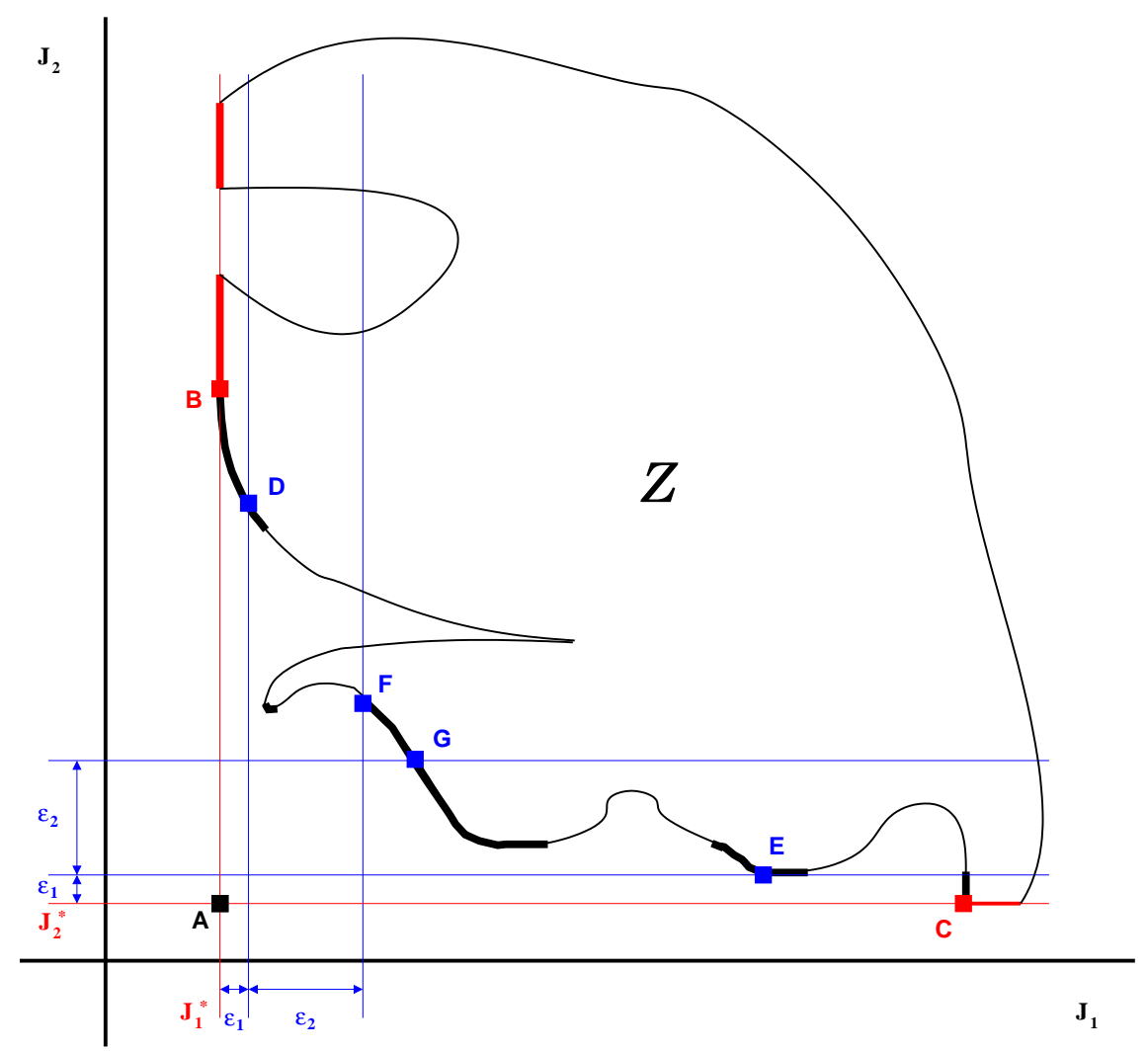

Figure 1. Graphical example of the Pareto front for a hypothetical optimisation problem with two criteria

Then, equation (10) is discretised using a sampling time $\Delta t=t_{n+1}-t_{n}$ where $t_{n+1}$ and $t_{n}$ are two consecutive time instants using an explicit numerical integration rule to approximate the above integral, as Euler or Runge-Kutta. For example, in case of using the Euler rule, the following equivalent discrete-time form is obtained:

$$
\vec{x}(k+1)=\vec{x}(k)+\Delta t \cdot \vec{f}(\vec{x}(k), \vec{u}(k), \vec{p})
$$

Once the problem is formulated as a NLP, it can be solved using a commercial optimisation software. In this paper, the General Algebraic Modelling System (GAMS) ${ }^{\mathrm{a}}$ is the optimisation package used to code and solve the NLP problem. The numerical optimisation method used to solve the problem is a generalised reduced gradient search, ${ }^{16}$ implemented in the NLP solver CONOPT $^{\mathrm{b}}$ available in the GAMS optimisation package, which can cater for the nonlinearities of the performance index and constraints.

The CONOPT optimisation algorithm starts by finding a feasible solution; then, an iterative procedure follows, which consists of:

- finding a search direction, through the use of the Jacobian of the constraints, the selection of a set of basic variables and the computation of the reduced gradient.

- performing a search in this direction, through a pseudo-Newton process until a convergence criterion is met.

A detailed description of the CONOPT algorithm and its implementation may be found in ${ }^{17}$ and in the manuals available at the GAMS web page.

\footnotetext{
ahttp://www.gams.com

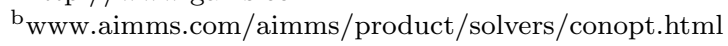




\section{Multi-objective optimisation}

A solution $\vec{z}^{*}$ of the multi-objective optimisation problem, presented in equation (1), is said to be Pareto optimal iff there does not exist another $\vec{z} \in \mathcal{Z}$ such that $J_{i}(\vec{z}) \leq J_{i}\left(\vec{z}^{*}\right)$ for all $i=1, \cdots, n_{j}$ and $J_{j}(\vec{z})<J_{j}\left(\vec{z}^{*}\right)$ for at least one index $j$. In other words, a solution is Pareto optimal if and only if an objective $J_{i}(\vec{z})$ can be reduced only at the expense of increasing at least one the other objectives. In general, there may be many Pareto optimal solutions to an optimisation problem.

Scalarisation is a common approach to solving such multi-objective optimisation problems and, therefore, a technique to choose a solution among all Pareto optimal ones. This means converting the problem into a single objective optimisation problem with a real-valued objective function, termed as the scalarising function, depending on some parameters. One of the most used strategies to obtain a scalar objective function is to form a linearly weighted sum of the functions $J_{i}$ :

$$
\min _{\vec{z} \in \mathcal{Z}}\left[J_{1}(\vec{z}), J_{2}(\vec{z}), \cdots, J_{n_{j}}(\vec{z})\right]=\min _{\vec{z} \in \mathcal{Z}} \sum_{i=1}^{n_{j}} w_{i} J_{i}(\vec{z})
$$

The priority of the objectives are reflected by the weights $w_{i}$, but choosing their value is not always a straightforward task. Although this type of scalarisation is widely used, it has serious drawbacks associated with it. ${ }^{18}$

In general, the most obvious problem with weighted formulae is the ad-hoc setting of the weights. This setting is based either on a somewhat vague intuition of the user about the relative importance of different quality criteria or in several trial and error experimentation with different weighting values. Another problem with weights is that, once a formula with precise values of weights has been defined, the optimisation algorithm will be effectively trying to find the best model for that particular setting of weights, missing the opportunity to find other solutions that might be actually more interesting to the user, and representing a better trade-off between different quality criteria. In particular, weighted formulae, involving a linear combination of different criteria, have the limitation that they cannot find solutions in a non-convex region of the Pareto front. ${ }^{18}$ This problem is particularly serious when the weighted formula involves a summation/subtraction (rather than a multiplication/division) of terms representing different magnitudes, often with very different scales in their units of measurement. This can be dealt by normalising the different quality criteria so that they refer to the same scale. This approach is well-known in the literature and at a first glance it is a very satisfactory approach. There is, however, a subtle problem associated with normalisation that is rarely discussed in the literature: in general there are several different ways of normalising, and the decision about which normalisation procedure should be applied tends to be ad-hoc as well.

\section{A. Lexicographic optimisation}

In the present work a different multi-optimisation technique is proposed with the purpose of avoiding the problems of the weighting approach based on lexicographic optimisation. This technique establishes a hierarchical order among all the optimisation objectives, named lexicographic order, from the most important $J_{1}$ to the least important $J_{n_{j}}$. A given $\vec{z}^{*} \in \mathcal{Z}$ is a lexicographic minimiser of equation (1) iff there does not exist a $\vec{z} \in \mathcal{Z}$ and a $j$ satisfying $J_{j}(\vec{z})<J_{j}\left(\vec{z}^{*}\right)$ and $J_{i}(\vec{z})=J_{i}\left(\vec{z}^{*}\right)$ for all $i=1, \cdots, j-1$. An interpretation of this definition is that a solution is a lexicographic minimum iff an objective $J_{i}$ can be reduced only at the expense of increasing at least one of the higher-prioritised objectives $\left\{J_{1}, \ldots, J_{(i-1)}\right\}$. Hence, a lexicographic solution is a special type of Pareto-optimal solution that takes into account the order of the objectives. This hierarchy defines an order on the objective function establishing that a more important objective is infinitely more important that a less important objective.

A standard method for finding a lexicographic solution is to solve a sequential order of single objective constrained optimisation problems. After ordering, the most important objective function is minimised, subject to the original constraints. If this problem has a unique solution, it is the solution of the whole multi-objective optimisation problem. Otherwise, the second most important objective function is minimised. Now, in addition to the original constraints, a new constraint is added to guarantee that the most important objective function preserves its optimal value. If this problem has a unique solution, it is the solution of the original problem. Otherwise, the process goes on iteratively. More formally, the lexicographic minimum of equation (1), lex $\min _{\vec{Z}} \vec{J}(\vec{z})$, can be found by using the following algorithm: 


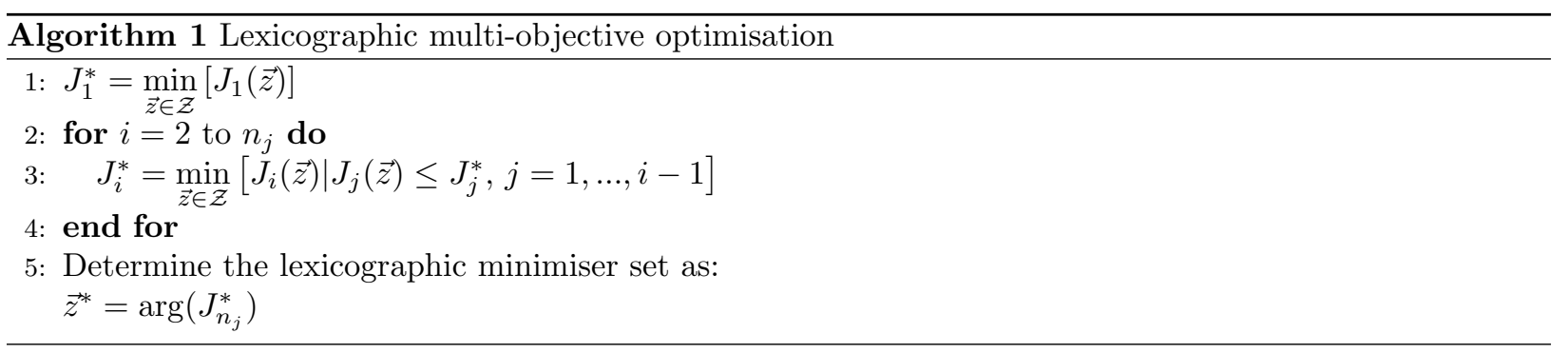

Lexicographic optimisation permits to sort a priori the different optimisation criteria according to its relative importance. This method has shown several benefits in front of the classical weighting methodology ${ }^{19,20}$ and has been started to be widely used in control engineering applications. ${ }^{21-23}$

\section{B. Establishing the lexicographic order}

In case the procedure designer in charge of publishing such a departure trajectory (i.e. the decision maker of this optimisation process) has a clear idea of what prioritisations should give to each location, maybe influenced by some political reasons, the use of the lexicographic approach is straighforward. In that case, previous algorithm leads to the best trajectory according to the desired hierarchy. However, in the case where this prioritisation is not clear, or when a more accurate scenario study is necessary, priorities should be inferred in some way.

One possible naive way, would consist in determining the prioritisation that optimises a performance index aimed at choosing the best trajectory among all the possibilities. One possible performance index is to select the prioritisation that minimises the maximum annoyance (equitable solution) in all the annoyance control points by running all optimisations considering all possibilities in the prioritisation order. More formally, let $J_{i}^{*}$ be the minimum annoyance that can be achieved at sensitive location $i$ (i.e. when location $i$ is in the first priority). Let $J_{i}^{P}$ be the annoyance at location $i$ reached with the optimal trajectory corresponding to priority $P$. For each priority $P$ a performance factor $\Delta^{P}$ can be defined as:

$$
\Delta^{P}=\max _{i}\left(J_{i}^{P}-J_{i}^{*}\right)
$$

Then, the best trajectory, $\vec{z}^{*}$ corresponds to the priority minimising this performance factor $\Delta^{P}$ :

$$
\vec{z}^{*}=\arg \left(\min _{P}\left(\Delta^{P}\right)\right)
$$

However, the number of different prioritisations is $n_{P}=n_{j}$ !, where $n_{j}$ is the total number of noise sensitive locations, what makes this approach prohibitive in real situation. Alternatively, a iterative approach can be used such that the lexicographic order is determined by fixing one by one the lexicographic priority orders of the objectives.

The following heuristic rule suggested by $\operatorname{Luss}^{24}$ can be used to determine dynamically the lexicographic order in which the objectives should be minimised: the first objective in the lexicographic order becomes that one that minimises the worst-case noise annoyance in any location. Once determined the first objective by solving an mono-optimisation problem using each time only one objective, this objective is fixed by imposing as a restriction that its value should always be less or equal to the optimal value found. Then, a set of mono-optimisation problems using the remainder objectives are run. From the results, the second objective in the lexicographic prioritisation minimised is fixed to the one that minimises the worst-case noise at the remaining locations maintaining the optimal level fixed for the first objective. This procedure is repeated iteratively until the lexicographic order of the whole set of objectives is set. As recognised in Ref. 24, this heuristic approach in general does not lead to the best solution (the equitable solution) provided by the exhaustive search approach, however the solution obtained is not far and provides a good compromise with respect to the computation time saved.

\section{Hierarchical lexicographic optimisation}

The previous lexicographic approach with priority discovering can be further elaborated by taking into account that using either the exhaustive lexicographic search approach or the heuristic method proposed in 


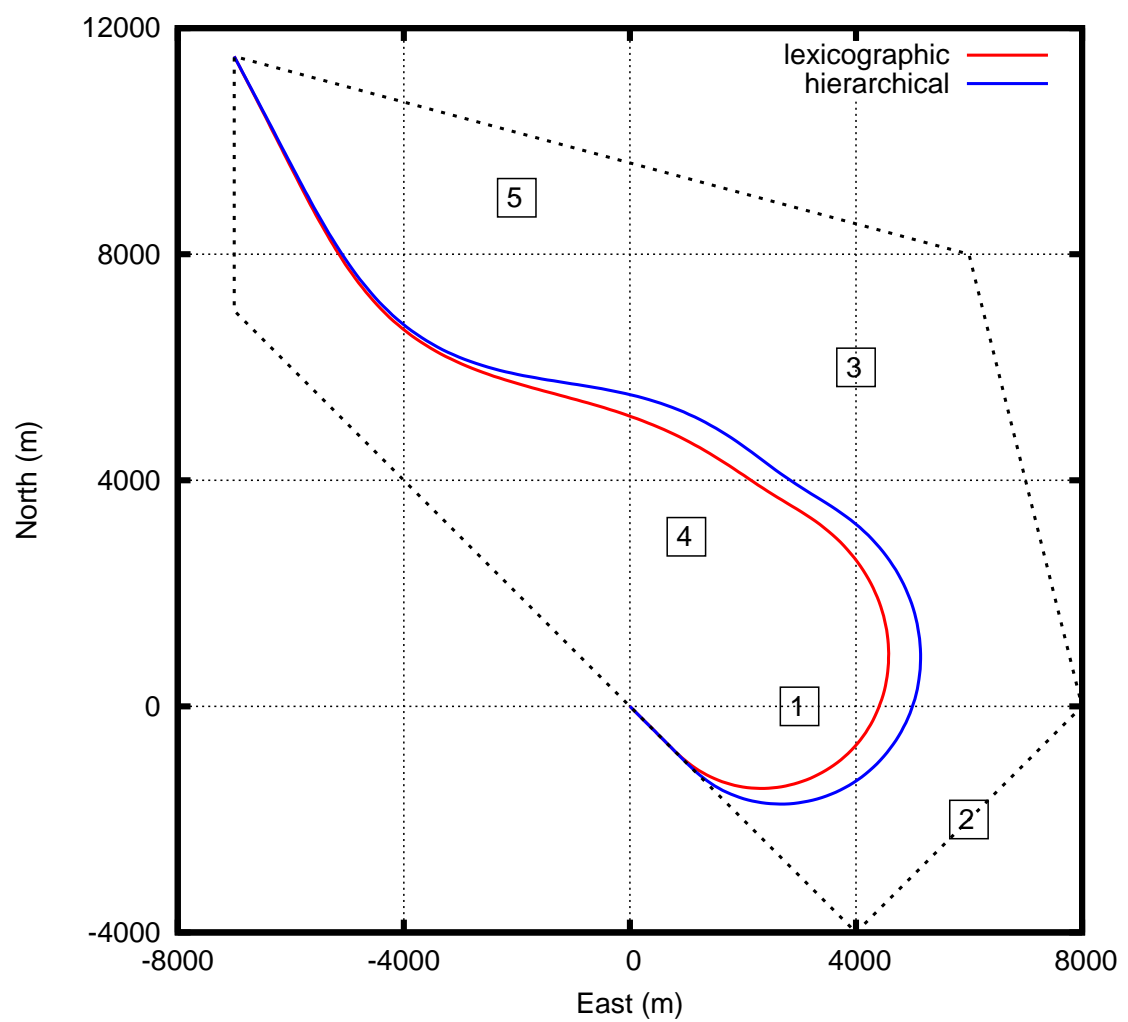

Figure 2. Horizontal track for the lexicographic and hierarchical optimal trajectories satisfying the equitable criteria

the previous section, there is no guarantee that in general the best solution based on the minimisation of the worst-case annoyance is achieved. This is due to using previous approaches only a subset of solutions of the Pareto optimal frontier are explored. To guarantee that the best solution is found the whole set of solutions in the Pareto optimal frontier should be considered. However, this is again prohibitive from the computational point of view. Alternatively, $\epsilon$-constraint method proposed in Miettinen ${ }^{18}$ can be used coupled to the exhaustive or heuristic lexicographic order search method. Such improvement consist in accepting an $\epsilon$ degree of relaxation in the optimal objectives fixed as a constraints in the lexicographic optimisation process. Varying the $\epsilon$ degree in a range the exploration of the Pareto optimal frontier is achieved and the best solution based on on the minimisation of the worst-case annoyance is achieved. Lexicographic optimisation using $\epsilon$-relaxation is know in the literature as hierarchical optimisation. To exemplify graphically the benefits of using this hierarchical approach the reader is referred to the Figure 1 where a simple case with only two optimisation criteria $\left(J_{1}\right.$ and $\left.J_{2}\right)$ is shown. This figure represents all the possible combination of values that both objective functions can take. The admissible set of solutions is enclosed in the area $\mathcal{Z}$. Point A represents the ideal or utopic solution, where the absolute minimum value for both objectives would be obtained. This point typically falls out of the admissible set of solutions and the decision maker has to choose a point among the infinite Pareto-optimal solutions, which in this figure are represented by a thick line. Red lines represent the mono-objective optimums for each criteria and when using a pure lexicographic approach, Pareto Optimal solutions B and $\mathrm{C}$ are obtained using, respectively $J_{1}$ and $J_{2}$ as the highest priority criteria. Points D and $\mathrm{F}$ shows that using $\epsilon$-relaxation (hierarchical optimisation) with two different $\epsilon$ better results for $J_{1}$ are obtained. Points $\mathrm{E}$ and $\mathrm{G}$ shows the same for objective $J_{2}$.

\section{Numerical example}

In this section, some results are shown that exemplify the method presented above applied to an hypothetical airport scenario where a departure route may be optimised. 


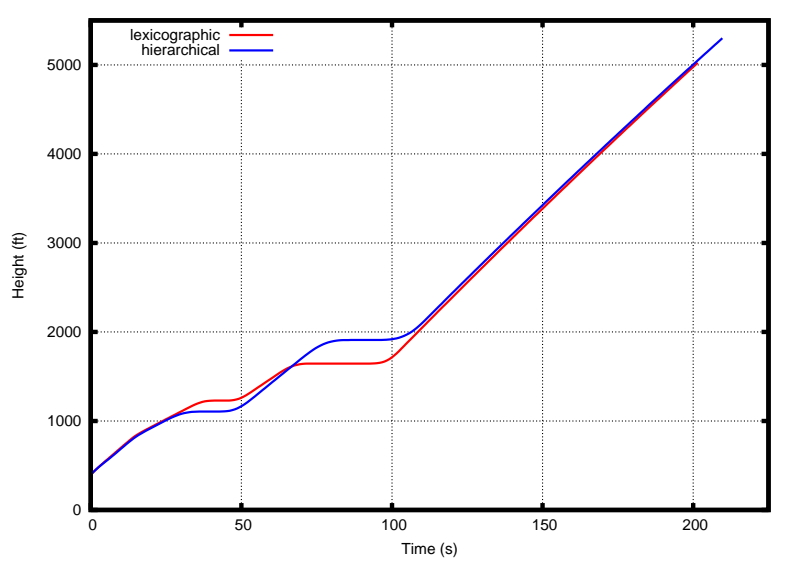

(a) Vertical profile

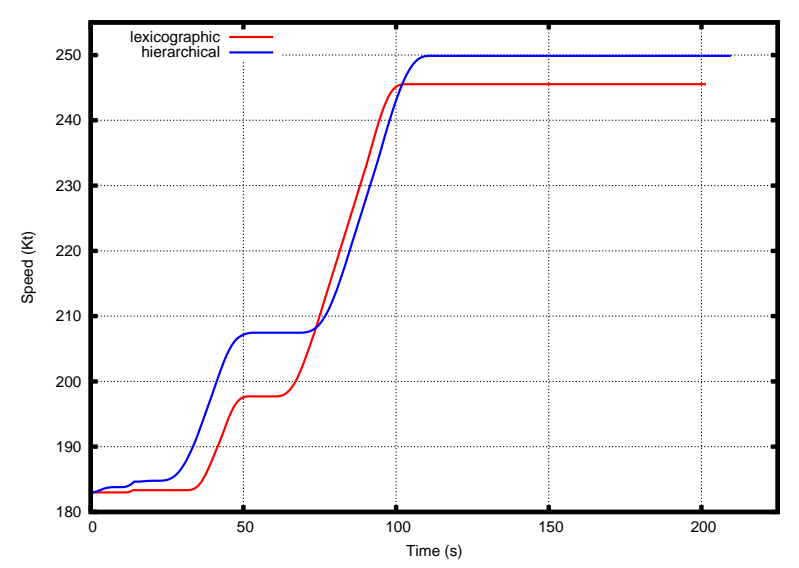

(b) Speed profile

Figure 3. Lexicographic and hierarchical optimal trajectories satisfying the equitable criteria

\section{A. Scenario description}

Table 1 summarises the data that define this scenario. In addition, being all trajectories below $10000 \mathrm{ft}$ maximum airspeed becomes $v_{\max }=250 \mathrm{Kt} .{ }^{1}$ The chosen aircraft model corresponds to the Airbus A340-600 equipped with Trent 556 engines and operating at its Maximum Take-off Weight, $(m=368000 \mathrm{~kg})$. Take-off is supposed to be performed with CONF3 flaps/slats configuration.

Table 1. Scenario definition data

\begin{tabular}{lr} 
Scenario parameter & Value \\
\hline Departing runway heading & $135^{\circ}$ \\
Minimum climb gradient & $3.3 \%$ \\
Initial point coordinates & {$[0,0] \mathrm{m}$} \\
Final point coordinates & $4000 \mathrm{ft}$ \\
Minimum height at final point & $10000 \mathrm{ft}$ \\
Maximum height at final point & 70 \\
Cost Index (CI) & 0.5 \\
Height Index (HI) & $0 \mathrm{kt}$ \\
Local wind & $50 \mathrm{~dB}(\mathrm{~A})$
\end{tabular}

Five different noise sensitive locations have been located in the vicinity of the departing runway. Table 2 contains the coordinates at each different noise sensitive location.

Table 2. Noise sensitive locations

\begin{tabular}{lrr} 
Sensitive location & North coordinate & East coordinate \\
\hline Location 1 & $0 \mathrm{~m}$ & $3000 \mathrm{~m}$ \\
Location 2 & $-2000 \mathrm{~m}$ & $6000 \mathrm{~m}$ \\
Location 3 & $6000 \mathrm{~m}$ & $4000 \mathrm{~m}$ \\
Location 4 & $3000 \mathrm{~m}$ & $1000 \mathrm{~m}$ \\
Location 5 & $9000 \mathrm{~m}$ & $-2000 \mathrm{~m}$
\end{tabular}




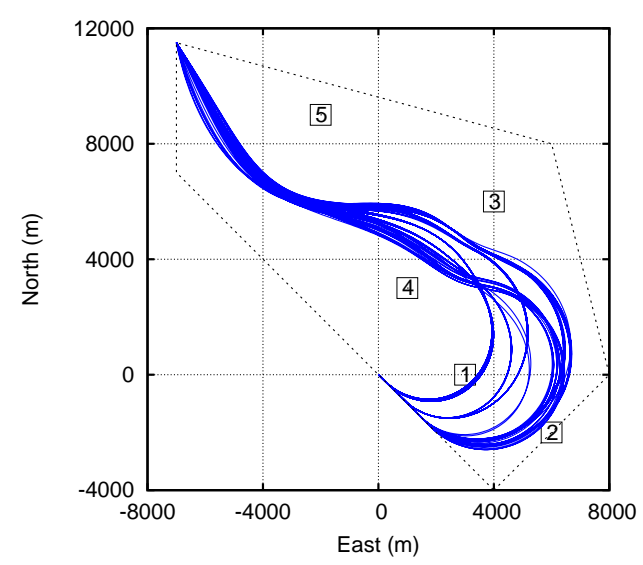

(a) $\epsilon=0 \%$ (Lexicographic case)

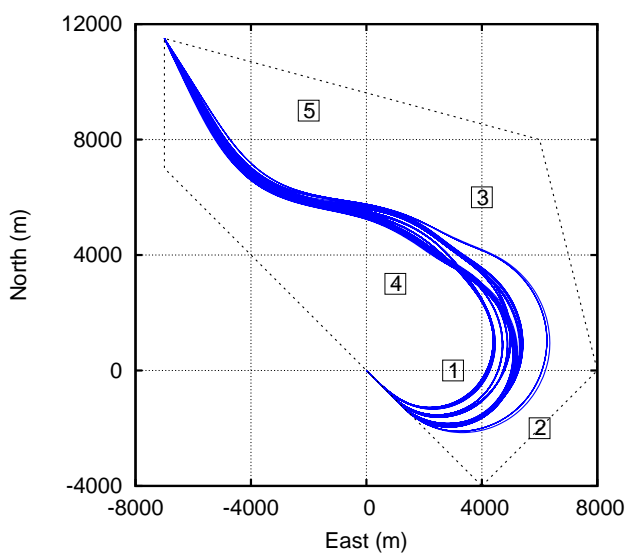

(c) $\epsilon=4 \%$

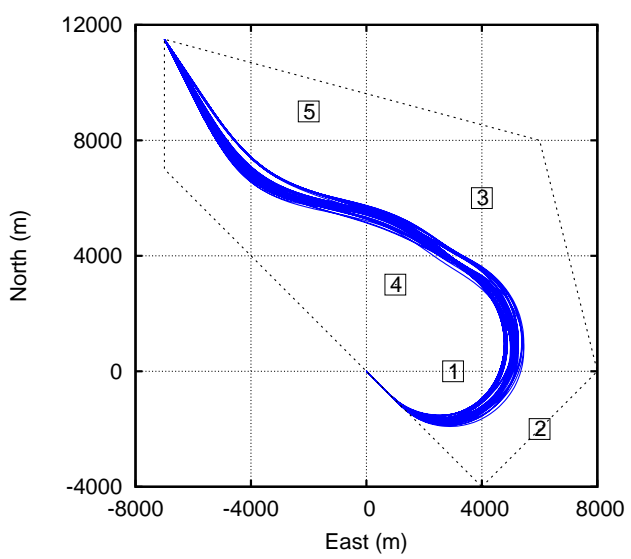

(e) $\epsilon=7.8 \%$

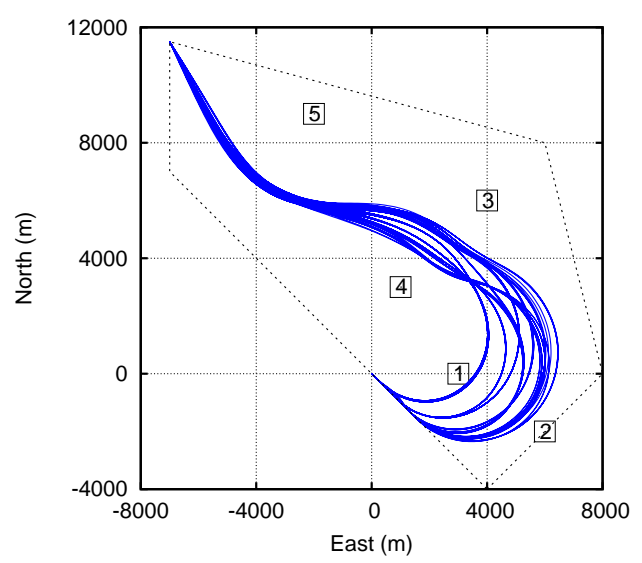

(b) $\epsilon=2 \%$

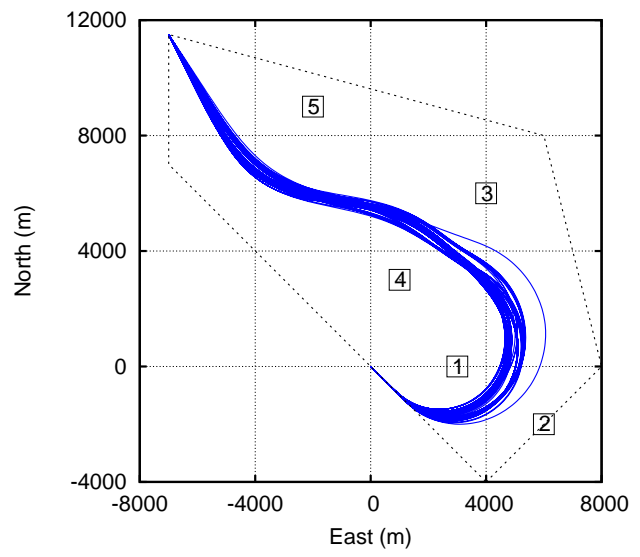

(d) $\epsilon=6 \%$

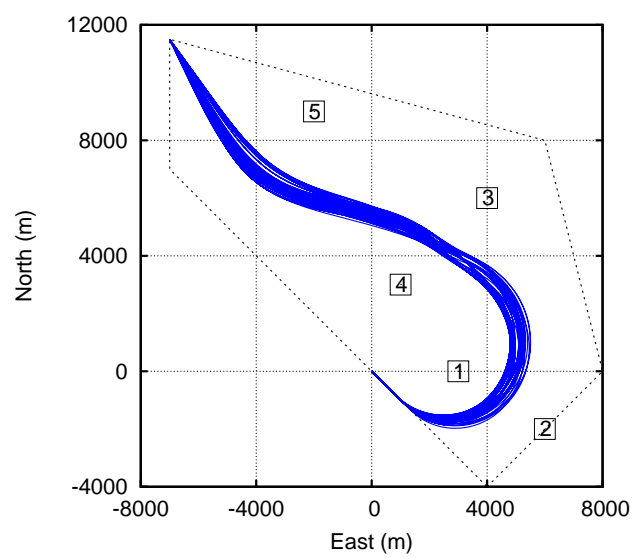

(f) $\epsilon=10 \%$

Figure 4. Optimal trajectories corresponding to all possible prioritisations in the lexicographic/hierarchical ordering

\section{B. Lexicographic solution}

In this example, the number of different noise sensitive locations is $n_{j}=5$ and the total number of different prioritisations is then $n_{j} !=120$. The lexicographic solution for this problem has been obtained independently by using either the exhaustive and heuristic methods described in section III.B. Algorithm 1 is slighly modified taking into account the effect of ambient noise: when the optimal value in a given location is lower 


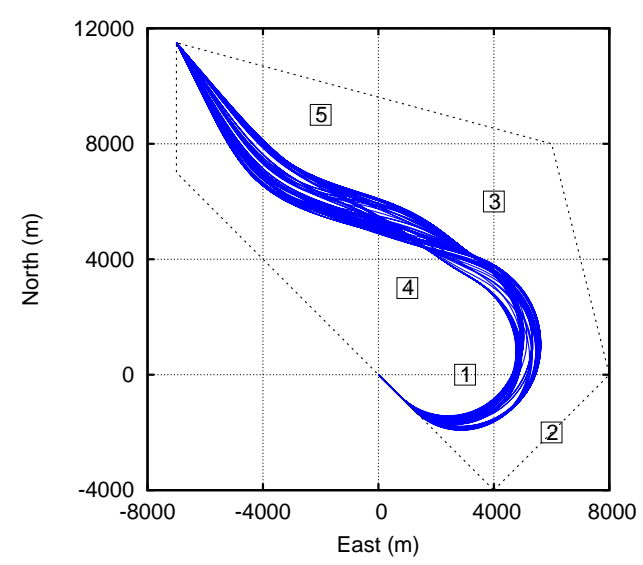

(a) $\epsilon=15$

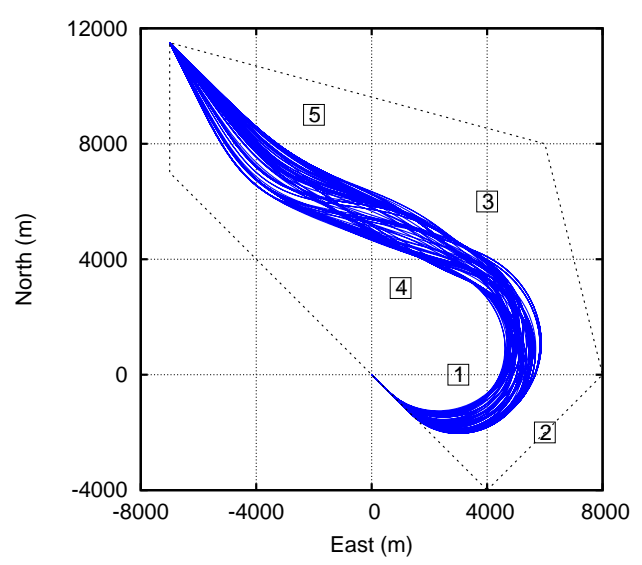

(b) $\epsilon=20$

Figure 5. Optimal trajectories corresponding to all possible prioritisations in the lexicographic/hierarchical ordering

than the ambient noise, noise restriction at this location becomes the ambient noise value itself. In addition, when algorithm 1 is finished, i.e. the 5 lexicographic steps are completed, an additional lexicographic optimisation is run minimising this time the airliner cost $C_{a}$ and maintaining all previous noise restrictions.

\section{Exhaustive lexicographic search}

Figure 2 shows, in red, the optimal lexicographic trajectory obtained for this particular scenario. This trajectory starts with a short straight segment continuing with the initial runway heading followed by a left turn. This turn produces a trajectory that avoids over-flying noise sensitive location number 2, producing a maximum noise level of $50 \mathrm{~dB}(\mathrm{~A})$ in this location. Being this value the ambient noise considered for this example, this means that this trajectory is not producing any noise disturbance at all at location number 2 . On the other hand, the maximum noise produced at first location is $65.2 \mathrm{~dB}(\mathrm{~A})$. The trajectory continues by passing someway in between locations 3 and 4, producing a maximum noise of $50.0 \mathrm{~dB}(\mathrm{~A})$ and $57.4 \mathrm{~dB}(\mathrm{~A})$ respectively. Finally, another curved segment allows the aircraft to fly far enough from location 5 producing $50.0 \mathrm{~dB}(\mathrm{~A})$ in this location.

Vertical and speed profiles for this trajectory are also shown, in figures 3 a) and b) respectively. The trajectory continues with the initial climb at $v_{2}=180 \mathrm{Kt}$ followed by a small acceleration segment starting at about $1230 \mathrm{ft}$. In this flat segment, speed reaches $198 \mathrm{Kt}$, allowing to retract the flaps and slats to CONF 2 configuration. Then, a second climb is started at constant speed until reaching $1650 \mathrm{ft}$ where a second acceleration segment takes place leaving the aircraft in CONF 1 configuration and accelerating to $245 \mathrm{Kt}$. At this constant speed, the final climbing segment takes place reaching $5000 \mathrm{ft}$ at the end of the trajectory.

In Table 3 are found the noise values at each location for this lexicographic optimisation trajectory. First line of the table contains the utopian set of optima, i.e. the absolute best noise values that can be achieved at each different location by running 5 independent mono-objective optimisations. In this particular case prioritisation 2-5-3-1-4 turns to be the most equitable solution according to equation (14). In this context, the worst location is location number 1 , where the difference between its utopian value and the actual value for this multi-objective trajectory is $10.7 \mathrm{~dB}(\mathrm{~A})$. Figure 4 a) shows all the 120 lexicographic optimal trajectories corresponding to the exhaustive search. As it can be seen, several prioritisations lead to the same final trajectory meaning that in some cases two or more prioritisations are equivalent.

\section{Heuristic method}

Prioritisation 1-2-3-5-4 is found as a result of the heuristic method, where the best lexicographic ordering is found iteratively. Table 3 contains the noise values at each location. As it can be seen, for this prioritisation the worst case is in location number 2 where the difference between the best one can achieve there and the actual value is $20.6 \mathrm{~dB}(\mathrm{~A})$. As expected, the solution obtained by using this heuristic method differs from the solution obtained by comparing all possible prioritisations. Prioritisation $1-2-3-5-4$ turns to be the 30 th 


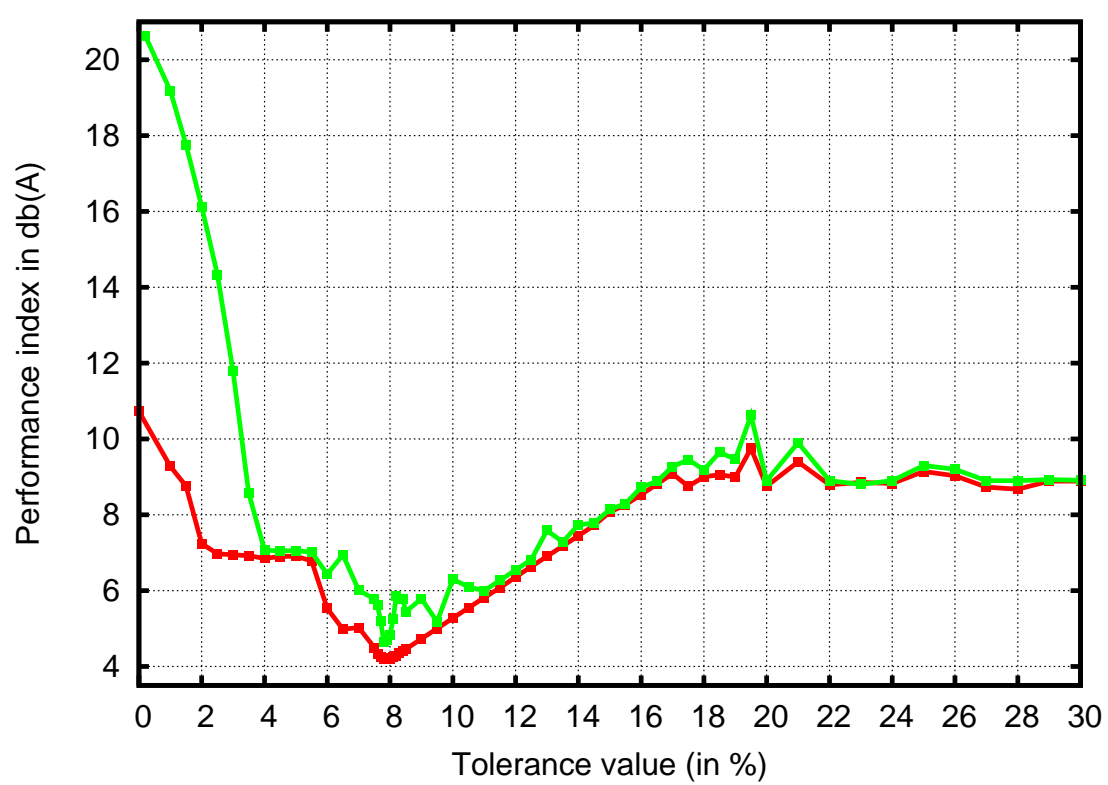

Figure 6. Best worst-case performance index $\left(\min _{P}\left(\Delta^{P}\right)\right)$ for the exhaustive and heuristic hierarchical optimisation optimal solutions in function of the tolerance value $\epsilon$

Table 3. Utopian and different multi-objective optimal values at all noise sensitive locations

\begin{tabular}{lrrrrrr} 
Optimisation method & $J_{1}$ & $J_{2}$ & $J_{3}$ & $J_{4}$ & $J_{5}$ & $\min _{P}\left(\Delta^{P}\right)$ \\
\hline Independent mono-objective (utopian) & 54.5 & 50.0 & 50.0 & 50.0 & 50.0 & - \\
Lexicographic-exhaustive $(\epsilon=0)$ & $\mathbf{6 5 . 2}$ & 50.0 & 50.0 & 57.4 & 50.0 & $\mathbf{1 0 . 7}$ \\
Lexicographic-heuristic $(\epsilon=0)$ & 54.6 & $\mathbf{7 0 . 6}$ & 57.1 & 55.3 & 50.0 & $\mathbf{2 0 . 6}$ \\
Hierarchical-exhaustive $(\epsilon=7.8 \%)$ & $\mathbf{5 8 . 7}$ & $\mathbf{5 4 . 2}$ & 54.0 & 54.3 & 50.0 & $\mathbf{4 . 2}$ \\
Hierarchical-heuristic $(\epsilon=7.8 \%)$ & 58.9 & $\mathbf{5 4 . 6}$ & 54.0 & 54.1 & 50.0 & $\mathbf{4 . 6}$
\end{tabular}

best trajectory among all 120 possibilities. As explained before, this method does not guarantee to find the best equitable solution, but the gain in computational cost is very significant being the sole practical methodology when $n_{j}$ is high (above 7 or 8). However the advantage of using the heuristic method is much more important when the hierarchical optimisation idea is applied, as it is explained in the next section.

\section{Hierarchical optimisation}

The same scenario presented above is solved now by using the hierarchical optimisation method, where the lexicographic technique is applied by allowing a relaxation of $\epsilon \%$ in the higher-priority constraints. In this example the exhaustive and heuristic methods have been tested for different values of $\epsilon$ ranging from $0 \%$ (pure lexicographic, as seen before) to $30 \%$ at regular intervals of $0.5 \%$. Figure 6 show, in red, the best worst-case performance index, according to equation (14), for each $\epsilon$ values. After a second round of optimisations, with smaller $\epsilon$ intervals, it turns that $\epsilon=7.8 \%$ provides with the best worst-case performance index, i.e. with the most equitable solution. Table 3 contains the noise values at each location for this optimal trajectory. This time, noise locations number 1 and 2 share the worst case, but thanks to the relaxation that the hierarchical optimisation technique introduces, the difference between the utopian al value at these locations and the actual values are only $4.2 \mathrm{~dB}(\mathrm{~A})$ in both cases. This is because the trade-off that exist now between all the optimisation criteria. Compared with the lexicographic method it can be seen that a small noise increase at locations 2 and 3 allows to reduce significantly the noise produced at location 1.

Figures 2 and 3 show, in blue, the resulting trajectory for this hierarchical optimisation. Finally, figures 4 a) to $\mathrm{f}$ ) and figure $5 \mathrm{a}$ ) and b) show all 120 possible prioritisations in function of different $\epsilon$ values. As it 
can be seen, as $\epsilon$ approaches its optimal value of $7.8 \%$ all different trajectories converge to a single one. This means that when moving around the optimal value all the trade-offs between the optimisation criteria that are established thanks to the $\epsilon$ tolerances lead to the same point in the Pareto front. In the two dimensional example shown in figure 1 that particular case would be when $\epsilon_{2}$ value in the figure leads that $F$ and $G$ points turn to be the same, meaning that the hierarchical solution is the same regardless of the prioritisation chosen. This behaviour is particularly advantageous when using the hierarchical heuristic method. As it was explained before, this method has the advantage of being much more computational friendly but it is not guaranteed that the most equitable solution is obtained by using it. When using the $\epsilon$ relaxations the convergence of all possible trajectories to a single one allows the solution obtained with the heuristic method to be closer to the actual solution. This behaviour can be seen in figure 6 where for each $\epsilon$ value the best worst-case performance index is plotted. Red values correspond to the exhaustive method and green ones to the heuristic method. As it can be seen, around the optimal value of $7.8 \%$ the difference between both methods becomes minimal (see Table 3).

\section{Conclusion}

Two approaches, based on lexicographic and hierarchical optimisation techniques, are presented in this paper. They are aimed at designing new noise abatement departure procedures where noise optimal departing routes are computed for a specific aircraft type and for a given scenario. Thus, a non-linear multi-objective optimal control problem is solved. First technique is an exhaustive search among all possible lexicographic or hierarchical solutions, requiring high computational costs. A second approach is presented by using an heuristic determination of the best lexicographic or hirerchical order. When pure lexicographic case is studied the heuristic approach does not guarantee the global optima. However, when the optimisation constraints are relaxed by a certain amount $\epsilon$ (i.e. hierarchical case) the convergence of all possible hierarchical trajectories to a single one allows the solution obtained with the heuristic method to be close to the actual solution. Therefore this combination of techniques (hierarchical and heuristic optimisation) seems to be a good and computational friendly approach to solve this kind of problems.

\section{References}

${ }^{1}$ ICAO, Procedures for Air Navigation Services - Aircraft Operations (PANS-OPS) - Volume I, Flight Procedures, International Civil Aviation Organisation (ICAO), Montreal (Canada), 4th ed., 1993, Doc. 8168-OPS/611.

${ }^{2}$ Prats, X., Nejjari, F., Puig, V., Quevedo, J., and Mora-Camino, F., "A framework for RNAV trajectory generation minimizing noise nuisances," 2nd International Congress on Research in Air Transportation (ICRAT), Belgrade (Serbia), June 2006.

${ }^{3}$ Prats, X., Quevedo, J., Puig, V., and Nejjari, F., "Planning of aircraft departure trajectories by using fuzzy logic and lexicographic optimization," The 2007 Congress and Exposition on Noise Control Engineering, Istanbul (Turkey), Aug. 2007.

${ }^{4}$ Visser, H. and Wijnen, R., "Optimization of noise abatement departure trajectories," Journal of Aircraft, Vol. 38, No. 4, July 2001, pp. 620-627.

${ }^{5}$ Visser, H. and Wijnen, R., "Optimisation of noise abatement arrival trajectories," The aeronautical journal, Vol. 107, No. 1076, 2003, pp. 607-615.

${ }^{6}$ Visser, H., "Generic and site spacific criteria in the optimization of noise abatement procedures," Transportation Research Part D: Transportation and Environment, Vol. 10, Sept. 2005, pp. 405-419.

${ }^{7}$ Wijnen, R. and Visser, H., "Optimal departure trajectories with respect to sleep disturbance," Aerospace Science and Technology, Vol. 7, 2003, pp. 81-91.

${ }^{8}$ Atkins, E. M. and Xue, M., "Noise-sensitive final approach trajectory optimization for runway-independent aircraft," Journal of Aerospace Computing, Information and Communication, Vol. 1, July 2004, pp. 269-287.

${ }^{9}$ Xue, M. and Atkins, E. M., "Noise-minimum runway-independent aircraft approach design for Baltimore-Washington international airport," Journal of Aircraft, Vol. 43, No. 1, Feb. 2006, pp. 39-51.

${ }^{10}$ Feng Zou, K. and Clarke, J.-P., "Adaptative real-time optimization algorithm for noise abatement approach procedures," AIAA's 3rd Annual Aviation Technology, Integration, and Operations (ATIO) Technology Conference, Vol. 1, Nov. 2003, AIAA paper No 2003-6771.

${ }^{11}$ Clarke, J.-P. and Hansman, R. J., "A systems analysis methodology for developing single event noise abatement procedures," Tech. rep., MIT Aeronautical Systems Laboratory, Cambridge, Massachusetts (USA), 1997, Report No ASL-97-1.

${ }^{12}$ EUROCONTROL, "Navigation strategy for ECAC," Tech. rep., European air traffic control harmonisation and integration programme, March 1999, Doc: NAV.ET1.ST16-001.

${ }^{13}$ FAA, Integrated Noise Model (INM) Version 6.0 Technical Manual, Office of Environment and Energy, Washington, DC. USA, Jan. 2002, FAA-AEE-02-01.

${ }^{14}$ Stevens, B. L. and Lewis, F. L., Aircraft control and simulation, John Wiley and Sons, INC., London, UK, 1992. 
${ }^{15}$ JAA, Joint Aviation Requirements. JAR-OPS: Airplane Operations, Joint Aviation Authorities (JAA), Hoofddorp (The Netherlands), 2003.

${ }^{16}$ Drud, A., "A GRG Code for Large Sparse Dynamic Nonlinear Optimization Problems," Mathematical Programming, Vol. 31, 1985, pp. 153-191.

${ }^{17}$ Drud, A., "CONOPT: A Large-Scale GRG Code," ORSA Journal on Computing, Vol. 6, 1992, pp. 207-216.

${ }^{18}$ Miettinen, K., Non-linear Multiobjective Optimization, Kluwer Academic Publishers, 1999.

${ }^{19}$ Marler, R. and Arora, J., "Survey of multi-objective optimization methods for engineering," Structural Multidisciplinary Optimization, Vol. 26, 2004, pp. 369-395.

${ }^{20}$ Srinivasan, B., Myszkorowski, P., and Bonvin, D., "A Multi-Criteria Approach to Dynamic Optimization," Proceedings of the American Control Conference, Seattle, Washington (USA), 1996.

${ }^{21}$ Aggelogiannaki, E. and Sarimveis, A., "Multiobjective constrained MPC with simultaneous closed-loop identification," International Journal of Adaptive Control and Signal Processing, 2008, (in press).

${ }^{22}$ Kerrigan, E. and Maciejowski, J., "Designing model predictive controllers with prioritised constraints and objectives," Proceedings of IEEE International Symposium on Computer Aided Control System Design, Vol. 1, 2002, pp. 33-38.

${ }^{23}$ Ocampo, C., Ingimundarson, A., Puig, V., and Quevedo, J., "Objective prioritization using lexicographic minimizers for MPC of sewer networks," IEEE Transactions on Control Systems Technology, 2008, (in press).

${ }^{24}$ Luss, H., "On equitable resource allocation problems: a lexicographic minmax approach," Operations Research Journal, Vol. 47, No. 3, June 1999, pp. 361-378. 